\title{
Philosophy of Chemical Medicines
}

\author{
Pijush Kanti Bhattacharjee
}

\begin{abstract}
This paper introduces an invention in working principle of Allopathic, Homeopathic, Ayurvedic, Herbal etc system of Medicines, with Vaccination, Physical Exercise, Yogic Exercise and Meditation etc. Development and use of Toxic substances from dead body of bad elements (Bacteria, virus, fungus, parasites etc) is very essence. This shows how minimum quantity of medicines recovers the diseases in a living being with the least side effects. It also identifies that by Physical Exercise, Yogic Exercise, Meditation etc, disease free condition can be prevailed. Perfect School of Medicines is designed keeping in view of medicinal as well as toxic substances effect derived from the dead bad elements.
\end{abstract}

Index Terms-Bad Elements-Bacteria, Viruses, Fungus, Parasites etc, Toxic substances, Perfect School of Medicines

\section{INTRODUCTION}

Every living body comprises with Good (Growth) substances and Bad (Toxic) substances. Good substances may be termed as healthy or growth substances (GS) consisting of Proteins, Vitamins, Minerals, Water etc. Bad substances are termed as poisonous or toxic substances (TS) containing bacteria, virus, fungus, parasites etc. These healthy or growth substances (GS) are gradually decreasing after facing death by a living body and finally it is converted into toxic substances (TS) only. We know that Bad Elements (bacteria, fungus, parasites, viruses etc), which cause disease in the living body, are also living creatures, because they can take part in reproduction. These type of disease caused bad elements like bacteria, parasites, viruses etc are searching the weaker section of the other living body, where they can be incremented i.e. dwell and reproduce for a period in their choiceable manner. These living bad elements are causing damage or disease to a living being. Our ultimate motto is to kill or throw out these living bad elements from the body of a living person by any means, generally by application of medicines or drugs which consist of chemical elements and compounds.

In every living body, there is immunity which safe guard the body from certain attacks of these bad elements like bacteria, fungus, parasites, viruses etc. These different types of immunology substances keep pace with the various parts of the body e.g. Spleen, Liver, Heart, Kidney and Brain etc. Whenever any of these immunology factors become less, that organ becomes weak and hence the organ is easily affected by the attack of bad elements (bacteria, fungus, viruses, parasites etc).

\section{LIVING BoDY AND DEAD BoDY}

When a disease affects one living body i.e. living body is attacked by bacteria or fungus or viruses or parasites etc and the disease is not cured within a specific time, that particular disease can cause death to the affected living body by increasing the amount of bad or toxic substances more than good or growth substances and ultimately remaining all good substances (GS) are converted to bad substances (BS) in a dead body. To be cure from the disease, we have to take help from medicines. All medicines i.e. allopathic or homeopathic or ayurvedic or herbal or any kind medicines are composed by chemical elements and compounds only. If medicines fail to cure i.e. to be free from bad elements (bacteria or fungus or viruses or parasites etc), we have to go for surgical treatment. Surgery is nothing, but to curtail the main affected part of the living body i.e. the main dwelling region of the so-called bad elements, causing damage to the organ. Most of the times it is seen that the surgical treatment is not the best method for complete cure e.g. Cancer, AIDs and Ulcer treatment etc. In Fig. 1, components present like GS and TS in a living and a dead body are shown explicitly.

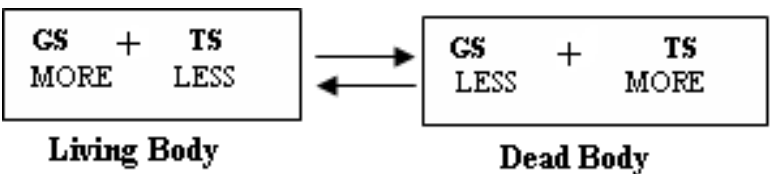

Fig. 1. Block diagram of living body and dead body with their contents. GS TS $\longrightarrow$ Good or Growth Substances Toxic Substances

\section{Allopathic, Homeopathic, Yogic Medicines WORKING METHODOLOGY AND IMPACT ON LIVING BEINGS}

Presently the most popular schools of medicines are Allopathic Medicine and Homoeopathic Medicine.

In Allopathic System, the significance (culture) of bad elements like bacteria, fungus, parasites, viruses etc are studied with different substances such as chemical compounds, antibiotics, tree products, herbals etc. Thereafter Medicines are selected or sensitive for the particular bad elements and finally the bad elements (bacteria, fungus, viruses, parasites etc) are killed or thrown out the affected living body by the application of certain amount of allopathic medicines. Generally we take the medicines in huge amount in allopathic system. The death of huge number bad elements (bacteria, fungus, viruses, parasites etc) produce large amount of toxic (poisonous) substances in the patient. These toxic substances cause ultimately damage to the other organs of the diseaseaffected body. Also the remaining living bad elements (bacteria, fungus, viruses, parasites etc) gather high immunity to the medicines due to withstanding in large amount of toxin and unused medicines, thus later on the disease appear more malignant (violent) in nature. Again the remaining and other associated parts of the medicines, which are not used for the killing or throwing out the bad 
elements, damage the organs of the patient. Hence the allopathic medicines are not considered as the best school of medicines; particularly it is named as side affected medicines. But the very essence of using this system is that it gives an immediate affect i.e. the patient is relieved within very short time, although he has to bear two side effects, namely one for unused medicines and the other for toxic substances released as a consequence of death or leaving of the bad elements (bacteria, fungus, viruses, parasites etc).

Ayurvedic system of medicines is working like Allopathic system. The only difference in Ayurvedic system is that the side effect due to excess medicines is little bit of less, since most of the medicines are derived from trees and herbal products which are also living beings. For that excess medicines act as user friendly with the patient, but the toxic substances evolved from the dead body of bad elements are remaining unused causing further side effects on the patient.

In Vaccination Theory, we introduce very small amount of the toxic substances derived from the dead bad elements (bacteria, fungus, viruses, parasites etc) to a living body for increasing immunity for a long time against the sudden attack of living bad elements. This works on the principle of killing or throwing bad elements (bacteria, fungus, viruses, parasites etc) from the living body by the help of toxic (poisonous) substances present in the bad elements either in living or dead condition.

In Homoeopathic System, on the other hand, very small amount of medicines (say $0.0005 \mathrm{mgm}$ i.e. few atoms daily) are applied. By that small amount of medicines, only a few numbers of bad elements (bacteria, fungus, viruses, parasites etc) can be killed or thrown out the diseased person. Let we consider only two number of living bad elements (bacteria, fungus, viruses, parasites etc) are killed by the introduction of homeopathic medicine, as a result the toxic substances will be produced from killing or dead body of the bad elements. Those toxic substances will take part for further killing or throwing the rest bad elements as chain reaction in a nuclear reactor. In Fig. 2. first homeopathic medicine which is applied to the patient kills two bad elements named BE1 and BE2, now total body of BE1 and $\mathrm{BE} 2$ are converted to toxic substances (TS), these toxic substances from dead BE1 and BE2 will further kill two living bad elements named BE3 and BE4, so bodies of BE3 and BE4 are converted to all toxic substances (TS) which can take part for further killing rest living bad elements in homeopathic system of medicines like chain reaction existing in a nuclear reactor or atom bomb blast.
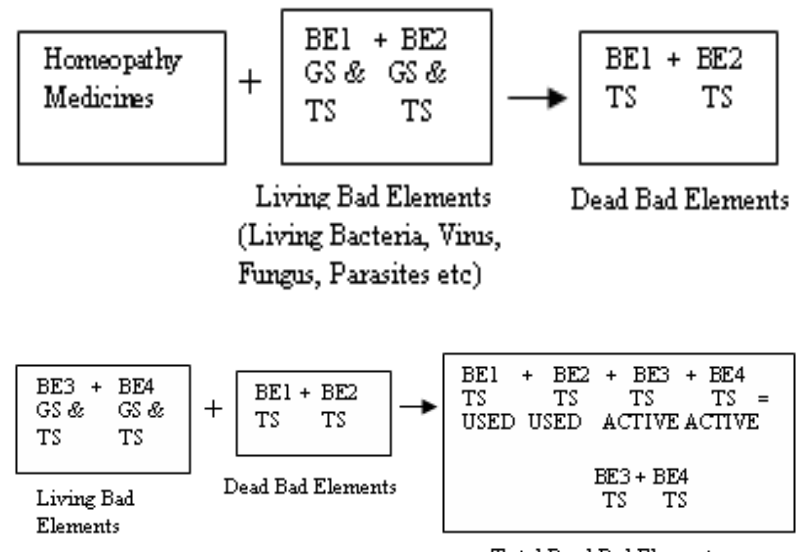

Total Dead Bad Elements
Fig. 2 Block diagram regarding activity of homeopathic medicines and toxic substances produced for killing or throwing out bad elements acting on the patient.

\section{ApPlication Philosophy FOR Allopathic, AYURVEDIC, HOMEOPATHIC, YOGIC MEDICINES}

In Homeopathic System, the medicines and the toxic substances produced by killing of the bad elements (bacteria, fungus, viruses, parasites etc) will take part jointly for further destruction of the living bad elements. It is same nature like the chain reaction happened in a nuclear bomb. Thus, the last killing bad elements will bring forth the death of next living bad elements and so on. Hence the residual toxic substances after death or throwing out the last number bad element (bacteria, fungus, viruses, parasites etc) remain very less, almost negligible amount and it cannot cause any malfunction of the other organs of the patient and also it acts as vaccine in future. This is a gradual or time consuming process, the patient has to wait for some time to get the effect of homoeopathic medicines, but in due course he will be completely cured. The main advantage of the application of homeopathic school of medicines is that actually very small amount of medicines i.e. chemical elements and compounds are required and there is no side effect either of medicines or toxic substances liberated by bad elements in the disease affected person. At the same time it has also very rare chance of reoccurrence of that particular disease in short time i.e. the disease is completely cured. The draw back of homeopathic system is to select the particular medicine for the disease according to the symptom of the patient and his surroundings, also the medicines are to be taken for a longer period till the last bad element killed or thrown. Sometimes the patient has to wait some period to get the full benefit. Overall homoeopathy school will assure the best result in medicinal field. The basic philosophy of Homeopathic Medicines, are described here above, will certainly indicate the actual working nature of homeopathic medicine in the living body. This theory (methodology) is invented first time. This homeopathy school of medicines has a complete difference with the working principle of allopathic medicines, which is also explained in detail. Thus in Allopathic system of medicine, patient has to bear two side effects, one due to excess amount of medicines (chemical elements and compounds) and the other is toxic substances produced due to throwing out or death of bad elements. Ayurvedic system is running on the same principal like allopathic system.

In Physical Exercise, Yogic Exercise and Meditation etc, we are killing or throwing out some bad elements (bacteria, fungus, viruses, parasites etc) by implying pressure and by producing more oxidation to blood called Oxy-hemoglobin, which enhances blood power. As a result toxic substances liberated, by the death or throwing out the few bad elements, take part for further killing or throwing out the rest bad elements like chain reaction and finally disease free condition is achieved.

\section{Perfecet School of Medicines Design}

We see that the great physicians like Dr. Hahnemann, Dr. Kent, Dr. Allen, Dr. Clarke, Dr. Ringer, Dr. Borick etc., all belonging to the allopathic system of medicines in their first 
life, turn to homeopathic school and develop different type of homeopathic medicines and applied methodology. They understand the urgent necessity of this area and they express that the disease can be completely cured by the application of homeopathic system of medicines only.

Lastly it should be our motto for the searching of the best school of medicines such that the disease can be cured completely with no further occurrence and causing the least side effects or nil side effects within a real time basis i.e. within a stipulated time. This is only possible in philosophy adopted by homeopathic school of medicine. I design a new school of medicines named as Perfect School of Medicines. Thus, Perfect School of Medicines is to optimize the medicines at each moment i.e. to determine the minimum quantity of requiring medicines in each moment before taking the medicines (or at least once in a day) in accordance with the living bad elements (bacteria, viruses, fungus, parasites etc) and toxic substances present due to killing or throwing the bad elements in the patient. Simply, we can conclude that the quantities of requiring medicines have to be decreased in accordance with the progress of recovery from the diseases for implementing Perfect School of Medicines philosophy.

\section{Conclusion And Advantage of Perfect School of MEDICINES}

The Perfect School of Medicines is the only way to recover the diseases completely with the least side effect in view of the excess medicines as well as toxic substances produced by the death or throwing out of the bad elements (bacteria, virus, fungus, parasites etc) in a disease affected living being. If you apply the Perfect School of Medicines philosophy to a patient and general Allopathic medicine philosophy to an another patient, when both the patients are affected by the same disease with equal intensity, it is observed that the patient imposing on Perfect School of Medicines is not only become cured (normal) within lesser time than that of the patient applied on Allopathic school philosophy, but have possessed more potential (energy) than the patient treated by Allopathic school. Moreover lot of bad elements (viruses, bacteria etc) can not be killed or removed from living body for want of proper medicines or treatment like Cancer, AIDs, Malignant Malaria, Asthma and other vulnerable diseases etc. It is seen that these types of diseases are causing due to numerous type of bad elements (viruses, bacteria etc), general medicines (antibiotics) can not be manufactured or cured for their treatment. By applying Perfect School of Medicines philosophy, these types of non curable diseases can also be cured within short times. Generally in Blood, Stool, Urine, Cells etc of the non-curable disease effected (like Cancer, AIDs, Asthma etc) living patient contain lots of disease causing living bad elements (viruses, bacteria, fungus etc), then the living bad elements are collected from the disposals (Stool, Urine, Plasma, Cell, Blood etc) of the patient and are killed either by high temperature and pressure treatment in a closed chamber or high voltage (electric shock) electrolysis or some chemical treatment. Then the dead body of the bad elements (viruses, bacteria etc) having toxic substances are pushed or drunk to the patient. The patient will be cured because of the living bad elements will be eliminated or dead by the toxic substances of the dead bad elements. Applying this method (system), no medicines are required to cure a patient from the affected dangerous diseases or bad elements.

By implementing this process, a new generating Philosophy of Medicines is explored. Here the requirement of the medicines is not only cut short, but at the same time a complete disease free condition is prevailed in living beings throughout the world. In this paper Philosophy of Chemical Medicines has been invented and explored in a new way.

\section{REFERENCES}

This is an innovation paper, no references is mentioned

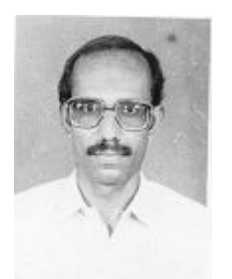

Dr. Pijush Kanti Bhattacharjee is associated with the study of Engineering, Management, Law, IndoAllopathy, Herbal, Homeopathic and Yogic medicines. $\mathrm{He}$ is having qualifications ME, MBA, MDCTech, AMIE, BSc, BA, LLB, BIASM, CMS, PET, EDT, FWT, DATHRY, BMus, KOVID, DH, ACE, FDCI etc. He worked in Department of Telecommunications (DoT), Govt. of India, as an Engineer from June 1981 to Jan 2007 (26 years), lastly holding Assistant Director Post at RTEC [ER], DoT, Kolkata, India. Thereafter, he worked at IMPS College of Engineering and Technology, Malda, WB, India as an Assistant Professor in Electronics and Communication Engineering Department from Jan, 2007 to Feb, 2008 and Feb, 2008 to Dec, 2008 at Haldia Institute of Technology, Haldia, WB, India. In Dec, 2008 he joined at Bengal Institute of Technology and Management, Santiniketan, WB, India in the same post and department. He has written two books "Telecommunications India" \& "Computer". He is a Member of IE, ISTE, IAPQR, IIM, ARP, India; CSTA, USA; IACSIT, Singapore and IAENG, Hongkong. His research interests are in Medicines Therapy, Image Processing, Quantum Computing, Wireless Communications, Network Security, Nanotechnology. 